\title{
Design and Research of Picking Manipulator Obstacle Avoidance System Based on IOT
}

\author{
https://doi.org/10.3991/ijoe.v14i03.8423 \\ Li Biqing \\ Hezhou University, Hezhou Guangxi, China \\ Tian Miao( $(\bowtie)$ \\ Henan University, Kaifeng Henan, China \\ $229292710 @ q q$.com \\ Zheng Shiyong \\ Wuhan University, Wuhan 430070, China \\ Ling Yongfa \\ Hezhou University, Hezhou Guangxi, China
}

\begin{abstract}
In recent years, wireless communication technology has developed rapidly and the speed of wireless communication data transmission is faster and faster, as well as remote control delay has greatly reduced, which is almost negligible, so the remote control is gradually being widely used in various fields. This paper achieves the motion control of picking manipulator and obstacle avoidance system with the advantages of IOT remote control technology and PLC real-time control system. Experiments show that the designed picking manipulator motion control and obstacle avoidance system not only have strong obstacle avoidance ability but also have smooth path optimization with high reliability and stability.
\end{abstract}

Keywords - Internet of Things; Remote Control; PLC; Manipulator; Motion Control; Obstacle Avoidance

\section{Introduction}

Robot and the Internet of Things remote control is the development direction of future modern agriculture instead of the traditional forest picking operations, improving the standardization of agricultural production and efficiency. Mechanical hand is an important part of the robot, and the control system programmed to achieve the desired operation, therefore, it reflects the strong artificial intelligence combining people and machines. This article will design and verify the motion control of manipulator and obstacle avoidance according to the characteristics of the picking robots and the operation mode of the manipulator so as to realize the obstacle avoidance and path optimization function of the manipulator. 


\section{Framework of Remote Motion Control System of Picking Manipulator}

This paper presents a remote motion control system based on PLC and includes client and server in order to realize the remote control of manipulator of picking robot, among which the server is divided into two parts, namely are video capture and manipulator control, and the client includes real-time monitoring, control, simulation and so on to the manipulator. The overall structure of the system shown in Figure 1.

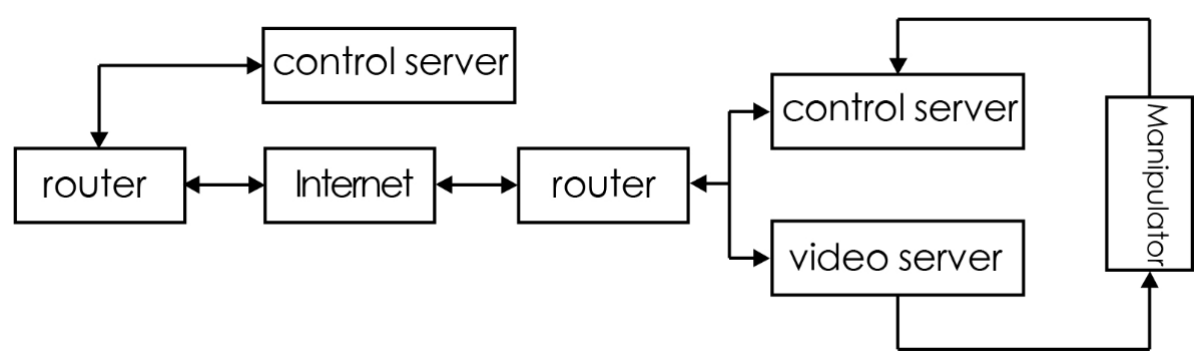

Fig. 1. Overall Frame Structure Chart of System

Shown in Figure 1, remote motion control system of picking manipulator consists of four parts, as follows:

1. Communication protocol module: a. The client and the server use network protocol to communicate. Specifying the format of the data packet on the client, and with a confirmation mechanism, while the server parses according to the data packet protocol; b. Determining the format of data transmission protocol packet of the visual server.

2. Robot-control server module: a. Client operates the interface of manipulator; $b$. simulation and obstacle avoidance of manipulator motion control; c. robot simulation system.

3. Manipulator module: Manipulator is used for picking and grabbing, is mainly responsible for the picking and grabbing of the target object.

4. Video server module: a. The front end of manipulator is equipped with CCD vision sensor, which is used to collect and obtain real-time information in front of it. The collected video is data analyzed and processed and then fed back to the client server.

Throughout the architecture, the manipulator is the most important module, which can achieve gripping action of claws according to the action of robot controlling server programming solenoid valve, the simulation model design shown in Figure 2. 


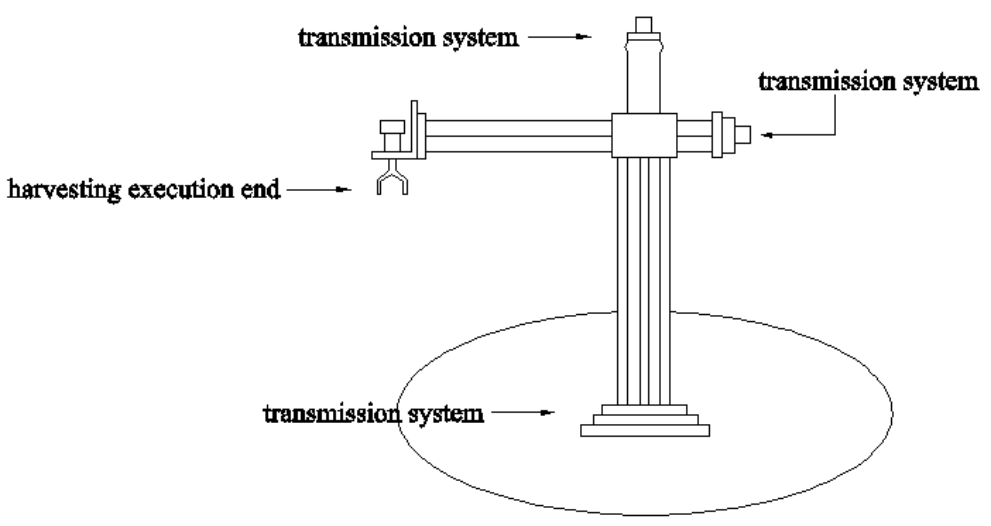

Fig. 2. Simulation Model of Picking Manipulator Module

\section{Hardware and Software Design of Motion Control And Obstacle Avoidance of Picking Manipulator}

\subsection{Hardware Platform of Picking Manipulator System}

PLC Control System. The design uses Simon s-200PLC as the core controller of the system, which contains the central microprocessor, memory, handheld programmer, power supply and various input and output interfaces. PLC controller usually adopts software programming to realize the function of relay, timer as well as digitalanalogue and digital conversion. In addition, PLC uses internal bus to realize the transmission of data information. The framework of PLC control system shown in Figure 3.

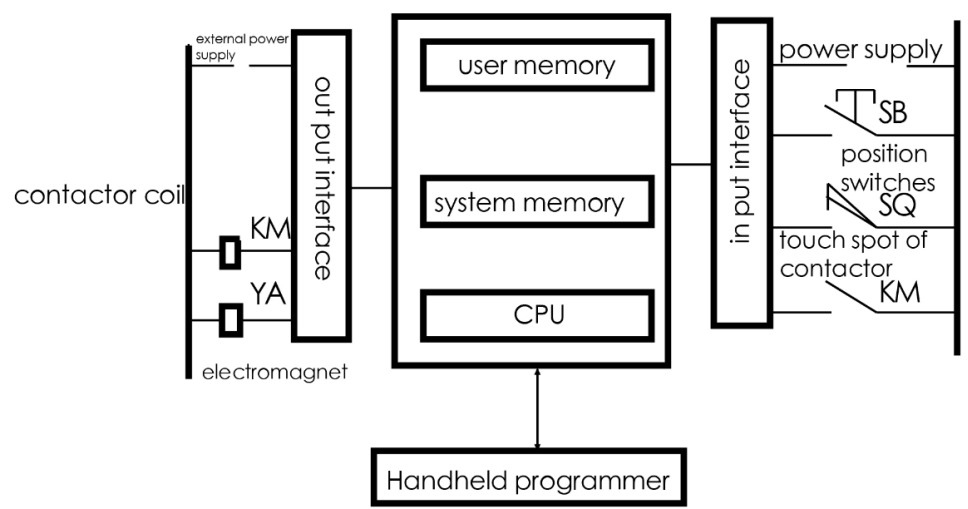

Fig. 3. Frame Diagram of PLC Control System 
Parameter Conversion of Manipulator Motion Control . When the system controls the manipulator, it needs to know the specific parameters such as its position, running speed and accelerated speed and so on in real time in the process of the manipulator motion control. In this paper, the control of the manipulator is controlled by the GT-400-SV motion control card. The units of each parameter are pulse, pulse/ST and pulse /ST 2 , among which ST is a cycle time to control it generating a pulse.

Set the exercise position. In the position setting of closed-loop servo system, the manipulator's speed and position parameters are expressed as (1) and (2) respectively:

$$
\begin{aligned}
& \text { position }=\frac{4^{*} p^{*} s}{L}(\text { pulse }) \\
& \text { position }=\frac{\mathrm{m}^{*} p^{*} s}{L}(\text { pulse })
\end{aligned}
$$

Where position is the movement position of the manipulator; $\mathrm{L}$ is the length of the manipulator joint.

Speed setting. In the closed-loop servo system, the manipulator speed is set to the speed control mode, the screw speed expression is:

$$
\frac{V(m / \mathrm{min})}{L(\mathrm{~mm} / \mathrm{r})}=\frac{V^{*} 10^{3}}{L}(r / \mathrm{min})
$$

Motor speed expression is:

$$
\frac{V^{*} 10^{3} * n}{L}(r / \min )
$$

According to the operating characteristics of PLC controller four times frequency, the expression of the servo controller setting the speed is:

$$
V \mathrm{el}=\frac{V^{*} n^{*} 4 p^{*} 10^{3}}{L}
$$

Among them, $\mathrm{V}$ is the motor speed; $\mathrm{Vel}$ is the setting speed of the servo controller.

Accelerated speed setting. In a closed-loop servo system, the drive is in speed control mode just like the previous two parameters, and the expression of the screw accelerated speed is:

$$
\frac{a\left(m / s^{2}\right)}{L(m m / r)}=\frac{a^{*} 10^{3} * n}{L}\left(r / s^{2}\right)
$$

And the motor speed calculation expression is: 


$$
\frac{a^{*} 10^{3} * n}{L}\left(r / s^{2}\right)
$$

According to the operating characteristic of PLC controller four times frequency, the expression of controller accelerated speed is:

$$
a c c=\frac{a^{*} n * 4 p * 10^{3}}{2}\left(\text { pluse } / s^{2}\right)
$$

Where aac is the acceleration value of controller setting; a is the motion accelerated speed of manipulator; $\mathrm{m}$ is the pulse multiplier.

\subsection{Software Platform of Picking Manipulator System}

Software design of picking manipulator system includes two aspects, namely are control server (lower computer) and the local client (PC). The control server mainly considers PLC as the core of the controller and is responsible for collecting the realtime operation status of the manipulator and analyzing the image information of the video server, as well as analyzing the data packet of the local server and controlling the manipulator in real time, and sending the data information to the local server side by the way of network communication to complete the interaction between the two messages. The local client is mainly composed of PC host computer control software, which can send control commands and receive and display the position, speed and accelerated speed information of picking manipulator. The software architecture of picking manipulator system as shown in Figure 4.

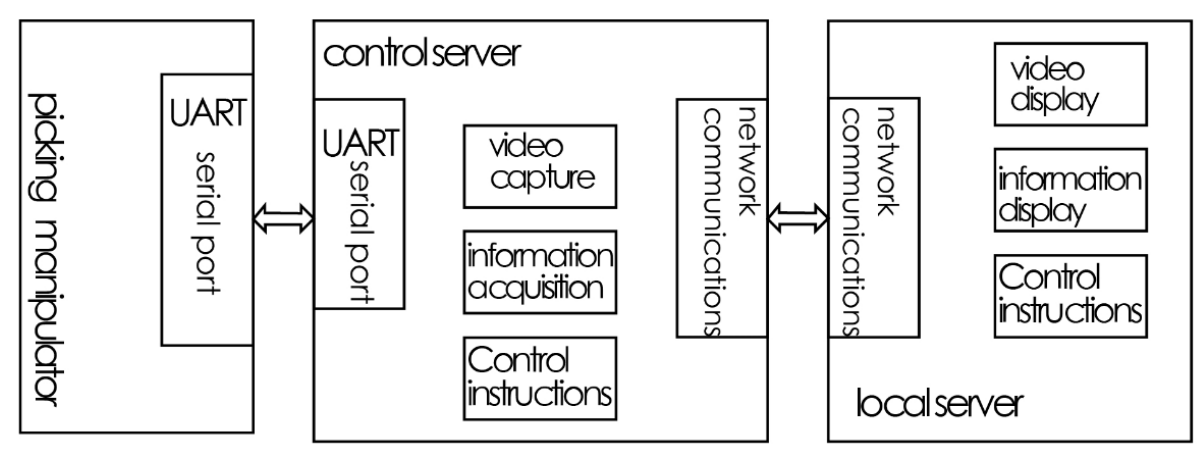

Fig. 4. Software Design Architecture of Picking Manipulator System

In the design of the whole software, the PLC control program in the control server is the most complicated, which includes four parts: public use, automatic, manual and initialization. Main program of PLC software shown in Figure 5. 


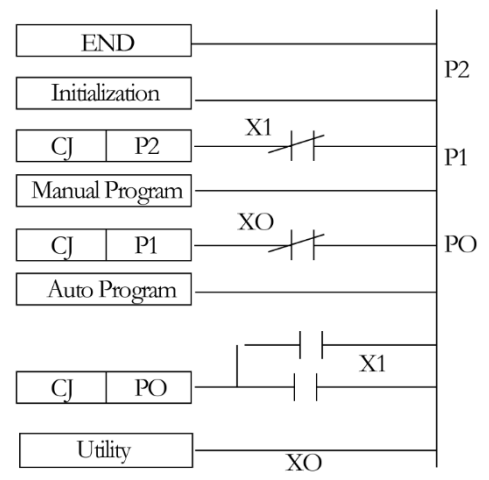

Fig. 5. Main Program Diagram of PLC Software

As shown in the figure above, $\mathrm{CJ}$ is a conditional jump instruction, which mainly implements program jump, reducing unnecessary execution time of the program and improving system efficiency. $\mathrm{X} 0$ and $\mathrm{X} 1$ are mode selection ports. When $\mathrm{X} 0$ is closed, and $\mathrm{X} 1$ is disconnected, the program is automatic control mode, and when $\mathrm{X} 0$ is off, and X1 is closed, the program is manual and back-to-the-original program.

\section{$4 \quad$ Kinematics And Obstacle Avoidance Principle of Picking Manipulator}

\subsection{Analysis of Dyn Motion of Picking Manipulator}

In order to realize the real-time control of the picking manipulator, we first need to set up the planar model of the manipulator, which is an important prerequisite for tracking and controlling the running trajectory of the manipulator. The plane mainly analyzes manipulator support structure dynamically through the mechanics principle, and achieves its control according to various parameters of the manipulator, achieving the purpose of path planning and obstacle avoidance. Plane and space structure of picking manipulator dynamics shown in Figure 6.

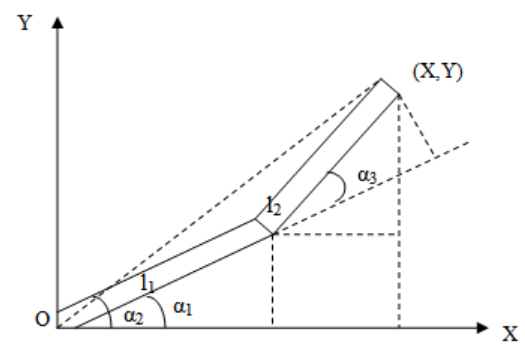

Fig. 6. Plane Structure Chart of Picking Manipulator Dynamics 


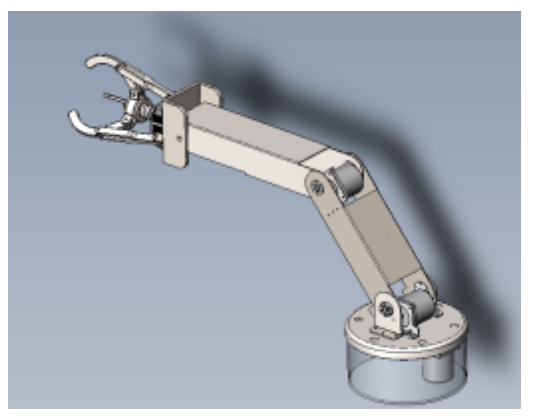

Fig. 7. Space Structure Chart of Picking Manipulator Dynamics

As shown in FIG. 7, 11 is an articulated rod 1 with a length of $250 \mathrm{~mm}$ and a variable angle of $0-180^{\circ} ; 12$ is an articulated rod 2 with a length of $250 \mathrm{~mm}$ and a variable angle of $0-100^{\circ}$. In addition, since the joint encoder of the manipulator adopts the encoder operation mode, the manipulator needs to be initialized each time the motion program is called. The expression of coordinate switch between joint coordinate system and rectangular plane coordinate system is:

$$
\left\{\begin{array}{l}
x=l_{1} \cos q_{1}+l_{2} \cos \left(q_{1}+q 2\right) \\
y=l_{1} \sin q_{1}+l_{2} \sin \left(q_{1}+q 2\right)
\end{array}\right.
$$

Where $\mathrm{x}$ and $\mathrm{y}$ are the coordinates of joint 2 in the rectangular coordinate system; and $\mathrm{q} 1$ and $\mathrm{q} 2$ are the angles of joint 1 turning in the spatial coordinate system.

The position of the end of joint 2 at the X-Y plane can be calculated according to Eq. (9), and then the motion of the manipulator can be controlled. The calculation expression is:

$$
\left\{\begin{array}{l}
r^{2}=x^{2}+y^{2} \\
\cos \alpha_{3}=\frac{l_{1}^{2}+l_{2}^{2}-r^{2}}{2 l_{1} l_{2}} \\
\sin \alpha_{3}=\sqrt{1-\cos \alpha_{3}^{2}} \\
\sin \alpha_{1}=\frac{l_{2} \sin \alpha_{3}}{r} \\
\cos \alpha_{3}=\frac{r_{1}^{2}+l_{1}^{2}-l_{2}^{2}}{2 l_{1} r} \\
\alpha_{1}=a \tan 2\left(\sin \alpha_{1}, \cos \alpha_{1}\right) \\
\alpha_{2}=a \tan 2(y, x) \\
\alpha_{3}=a \tan 2\left(y-l_{1} \sin q_{1}, x-l_{1} \cos q_{1}\right)
\end{array}\right.
$$

We can get the expression of inverse kinematics based on (9) and (10) : 


$$
\left\{\begin{array}{l}
q_{1}=\alpha_{2}-\alpha_{1} \\
q_{1}=\alpha_{3}-\alpha_{1}
\end{array}\right.
$$

The control server directly processes the joints of the manipulator. When controlling the manipulator, the distance traveled by the manipulator and its current position need to be calculated, and the calculation of the distance is usually performed by using a pulse, and the calculation expression is as follows:

$$
\text { position }=\frac{m^{*} p^{*} s}{L}(\text { pulse })
$$

Where $\mathrm{s}$ is the absolute distance from the articulation to the target position.

The distance is calculated as:

$$
s=\frac{\beta * 2 \pi * l}{360}
$$

Where $\beta$ is the angle which manipulator joint 2 turns.

\subsection{Obstacle Avoidance Principle of Picking Manipulator}

A tangent or overlap of a manipulator and an obstacle indicates the possibility of a collision, otherwise, the probability of a collision is zero. Therefore, obstacle avoidance can be achieved by only considering the situation where the manipulator and the obstacle target are tangent or overlapped in the process of manipulator motion control. In order to determine the possibility of collision between manipulator and obstacle, this paper uses artificial potential field method to analyze the surface repulsion of manipulator and obstacle, and dsafe and dmin respectively represent the safety distance and limit distance from manipulator to obstacle; $d$ is the minimum distance from manipulator to obstacle surface. The distance relationship between manipulator and the obstacle surface is shown in FIG. 8.

As shown in Figure 8, repulsive force is calculated according to three direct distances, respectively from $\mathrm{d}$, dlim, dsafe to manipulator:

$$
f_{v}=\left\{\begin{array}{cc}
0 & d>d_{\text {safe }} \\
K\left(\frac{1}{d}-\frac{1}{d_{\text {safe }}}\right) & d_{\text {lim }}<d<d_{\text {safe }} \\
f_{\max } & d<d_{\text {lim }}
\end{array}\right.
$$

Where $\mathrm{K}$ is the proportional coefficient; fmax is the maximum repulsion. The relationship between $\mathrm{K}$ and fmax is: 


$$
K=f_{\max } \frac{d_{\text {safe }}-d_{\text {lim }}}{d_{\text {safe }} d_{\text {min }}}
$$

$\mathrm{D}$ is less than dsafe when the manipulator is near to the obstacle, indicating that there is a possibility of collision. When the manipulator continues to move to obstacle, that is, when $\mathrm{d}$ is smaller than dlim, indicating that the system is in an emergency, and if the manipulator does not change the route, collide happens immediately. In this case, in order to prevent it from happening outside, you should stop manipulator moving to the obstacle direction. Instead, you should change the route to avoid obstacles so as to make manipulator into safe environment.

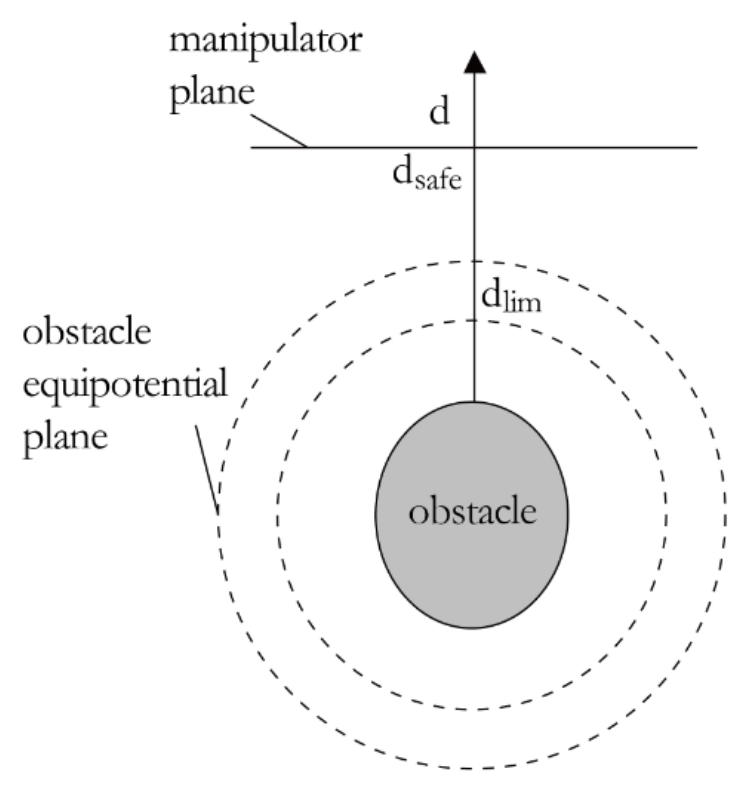

Fig. 8. Distance from Manipulator to Obstacle Surface

\section{$5 \quad$ Analysis of Experiments and Results}

In order to verify the real-time nature and reliability of motion control and obstacle avoidance system of picking manipulator, this paper used MATLAB to simulate the experiment. In the test, the step length was set to $0.5^{\circ}$, and the purpose of the test was to test whether the manipulator had the ability to effectively avoid obstacles to the space obstacle and re-route. Simulation results shown in Figure 9. 


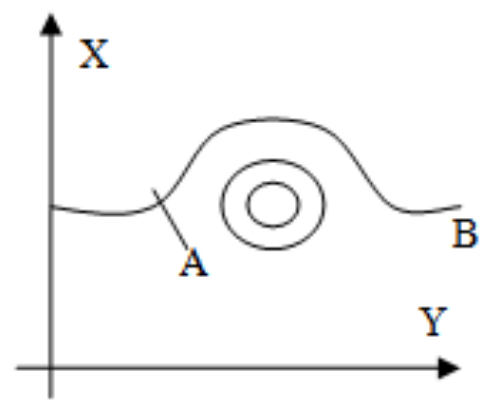

Fig. 9. Simulated Chart of Manipulator Obstacle Avoidance

As shown in FIG. 9, the manipulator advanced in the positive direction of the $\mathrm{x}$ axis and reached the point A when stepped 180 times. At this moment, the maximum repulsive force fmax was reached with an obstacle. Therefore, the system adopted the obstacle avoidance measure and the obstacle was successfully bypassed. The experimental results showed that the system can avoid the obstacle to the target point with the optimal arc path when it encountered the obstacle, and the optimization effect was obvious with strong obstacle avoidance and path planning ability.

\section{Conclusion}

In this paper, the frame of remote motion control system of picking manipulator is described firstly. Then the whole hardware and software of the system are designed effectively. Finally, the motion process of the manipulator is modeled, the principle of motion control and obstacle avoidance is calculated and analyzed, and motion control and obstacle avoidance system of picking manipulator have been realized. The experimental results showed that the system can avoid the obstacle to the target point with the optimal arc path when it encountered the obstacle, and the optimization effect was obvious with strong obstacle avoidance and path planning ability.

\section{$7 \quad$ Acknowledgment}

This work is supported by the following fund:

2016 The project of improving the basic ability of young teachers in Colleges and universities in Guangxi:"Design and development of electronic commerce platform of agricultural products based on Semantic Technology"(No,KY2016YB455).

2015 college students' innovative training program: "Research on the application of value added travel experience in the mobile terminal of the 'ethnic custom travel' in Guangxi” (No 201511838070);\&\& “The design and development HeYuanTong Campus Mobile Phone APP based on Android" (No 201511838034).Project of scientific research and technology development project of Hezhou: "Design and implementation of agricultural products e-commerce platform based on Semantic Technology" (No,Hekeneng 1506006). 


\section{$8 \quad$ Reference}

[1] B.Q LI, Y.F LING, H.Y ZHANG, S.Y ZHENG: The Design and Realization of Cherry Tomato Harvesting Robot Based on IOT. International Journal of Online Engineering, 12(12), 23, (2016). https://doi.org/10.3991/ijoe.v12i12.6450

[2] B.Q LI, W.L GUAN, S.Y Zheng, X.G Yue: OPTIMISATION DESIGN OF CORN PRECISION SEEDER BASED ON MULTI-ROUTE AND MULTI-CHANNEL CONTROL. Journal of The Balkan Tribological Association, 21(4A), 1215, (2015).

[3] U. A. SHAABAN, M. T. AFIFY, G. E. HASSAN, Z. A. EL-HADDAD: Development of a Vacuum Precision Seeder Prototype for Onion Seeds. Misr J. Ag. Eng, 26(4), 1751, (2009).

[4] B.Q LI, X.M YANG GUAN, S.Y ZHENG : Internet of Things-based Simulation Study on Lijiang River Water Environment Monitoring. Journal of Coastal Research,79, 1-5, (2017).

[5] Y. A. SHAABAN: Development of an Appropriate Pneumatic Planter for Small Holding in Egypt. Colg Agric, Bha Uni, 46(8), 1999, (2010).

[6] P. K. AGARWAL, J. BASCH, L. J. GUIBAS, J. HERSHBERGER, L. ZHANG: Deformable Free Space Tiling for Kinetic Collision Detection. Proc. 4th Wsp Alg Fd. Rbt, 54(3), 245, (2000).

[7] P. K. AGARWAL, J. ERICKSON, L. J. GUIBAS: Kinetic Binary Space Partitions for Intersecting Segments And Disjoint Triangles. Proc. 9th ACM-SIAM , 44(6), 578, (1998).

[8] Z.B. BARUT, A. OZMERZI: Effect of Different Operating Parameters on Seed Holding in the Single Seed Metering Unit of a Pneumatic Planter. Turk. J. Agric, 28, 435, (2004).

[9] M. J. ATALLAH: Some Dynamic Computational Geometry Problems. Comp. Math. Appl, 11(12), 1171 (1985). https://doi.org/10.1016/0898-1221(85)90105-1

[10] J. BARRAQUAND, J. C. LATOMBE: Robot Motion Planning: A Distributed Representation Approach. Int. J. Rbt. Res, 10, 628, (1991). https://doi.org/10.1177/0278364991 $\underline{01000604}$

[11] M. BERN, P. PLASSMANN: Mesh generation. In J.-R. Sack and J. Urrutia, editors, Handbook of Computational Geometry. Els Sci Pub B.V. Ams, 16(4), 566, (2000). https://doi.org/10.1016/B978-044482537-7/50007-3

[12] A. VASS-VARNAI, R. BORNOFF: Thermal Simulations and Measurements - a Combined Approach for Package Characterization. ICEP, Jpn,76(4), 886, (2000).

[13] V. THIYAGARAJAN, K. KALAICHELVAN, K.SRINIVASAN,S. VENUGOPAL, R. VIJAY: Influence of Specific Heat Capacity on Hybrid Non-asbestos Brake Pad Formulation. J. Balkan Tribol. Asc, 10(8), 102, (2015).

[14] L. YANG, X.T. HE, T. CUI, D. X. ZHANG, S. SHI, R. ZHANG, W. MANTAO: Development of Mechatronic Driving System For Seed Meters Equipped on Conventional Precision Corn Planter. Int J Agric \& Biol Eng, 8(4), 1, (2015).

[15] D. KARAYEL, Z.B. BARUT, A. OZMERZI: Mathematical Modeling of

[16] Vacuum Pressure on a Precision Seeder. Byst. Eng, 87(4), 437, (2004).

[17] X.G. YUE, G. ZHANG, Q. WU, F. LI, X.F. CHEN, G.F. REN, M. LI: Wearing Prediction of Stellite Alloys Based on Opposite Degree Algorithm. Rare Metals, 34(4), 125, (2015). https://doi.org/10.1007/s12598-014-0430-0

[18] Z. ZONGMING: Research on Wearing Prediction of the Cylinder Surface Based on Time Series Autoregressive (AR) Model. ICMTMA, Hong Kong, 12(1), 960, (2013). https://doi.org/10.1109/ICMTMA.2013.239

[19] S. SPINU: Numerical Simulation of Viscoelastic Contacts. Part 1. Algorithm Overview. J. Balkan Tribol. Assoc, 10(5), 269, (2015). 


\section{Authors}

Li Biqing (1984-), comes from Nanning, Guangxi province and is with the School of Information and Communication Engineering, Hezhou University, Hezhou Guangxi 542899, China. Master degree, senior engineer, the main research direction for Mobile terminal broadband MINO, IOT.

Tian Miao (1984-), comes from Zhengzhou, Henan province and is with the School of Software, Henan University, Kaifeng Henan 475004, China. Bachelor, assistant experimentalist, the main research direction for the Internet of Things. (229292710@qq.com).

Zheng Shiyong (1983-), comes from Guilin, Guangxi province and is with Wuhan University, Wuhan 430070 , China. $\mathrm{PhD}$ student, senior engineer, the main research direction for network marketing, IOT.

Ling Yongfa (1973-), come from Shangyou, Jiangxi province and is with the School of Information and Communication Engineering, Hezhou University, Hezhou Guangxi 542899, China. PhD, professor, the main research direction for Wireless communication network, IOT.

Article submitted 11 February 2018. Final acceptance 05 March 2018. Final version published as submitted by the authors. 